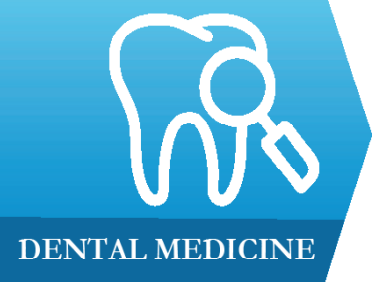

1) Department of Restorative Dentistry, Faculty of Dentistry, Marmara University, Istanbul, Turkey

2) Department of Conservative Odontology, Iuliu Hatieganu University of Medicine and Pharmacy, Cluj Napoca, Romania

3) Department of Restorative Dentistry, Faculty of Dentistry, Istanbul University, Istanbul, Turkey

DOI: $10.15386 / \mathrm{mpr}-1973$

Manuscript received: 17.11 .2020

Received in revised form: 06.07.2021

Accepted: 26.07.2021

Address for correspondence:

bora.korkut@marmara.edu.tr

This work is licensed under a Creative Commons Attribution-NonCommercialNoDerivatives 4.0 International License

\title{
Effect of surface sealants on color stability of different resin composites
}

\author{
Bora Korkut ${ }^{1}$, Marius Bud ${ }^{2}$, Pelin Kukey ${ }^{1}$, Hande Sar Sancakli ${ }^{3}$
}

\begin{abstract}
Objective. The aim was to assess the effect of surface sealants on the color stability of different composite materials.

Methodology. 120 samples were prepared using Filtek Ultimate (3M, USA), Charisma Topaz (Kulzer, Germany), and Clearfil Majesty Es-2 Premium (Kuraray Noritake, Japan) composites. The samples were polished with polishing discs and divided by test groups ( $\mathrm{n}=10$ for each), regarding the sealant used; Permaseal (Ultradent Products, USA), Biscover LV (Bisco, USA), Optiglaze Clear (GC Corp., Japan) and control. The samples were discolored for 144 hours in coffee solution at constant $37^{\circ} \mathrm{C}$, renewing daily. Color measurements were performed at the baseline, following the sealant application, and after discoloration procedure, using a spectrophotometer (EasyShade 5, Vita-Zahnfabrik, Germany). The collected $\mathrm{L} * \mathrm{C} * \mathrm{~h} *$ coordinates were converted to corresponding $\mathrm{L} * \mathrm{a} * \mathrm{~b} *$ coordinates and the color changes $(\Delta \mathrm{E})$ were calculated.

Results. All the sealants presented perceptible $(>1.2)$ and unacceptable $(>2.7)$ color changes. Biscover LV presented the lowest $\Delta \mathrm{E}$ among all sealants $(p<0.001)$. The highest $\Delta \mathrm{E}$ was observed for Permaseal $(p<0.001)$. Color changes for Optiglaze and control groups were similar $(\mathrm{p} \geq 0.05)$. Filtek Ultimate presented the highest color stability $(p<0.001)$, followed by Charisma Topaz and Clearfil Majesty. The combination of Filtek Ultimate with Biscover LV was considered to have the highest color stability, whereas the combination of Clearfil Majesty with Permaseal was considered the lowest, among all.
\end{abstract}

Conclusion. Experimented sealants were considered relatively effective on color stability of resin composites depending on the type of the sealant and the composite material. However, the use of surface sealants may not be advantageous every time. Biscover LV have inhibited the level of discoloration, whereas Permaseal had an increasing effect generally.

Keywords: sealant, Biscover LV, Optiglaze, Permaseal, discoloration, surface staining

\section{Introduction}

Increasing esthetic expectations of patients have increased the importance of the color match and longterm color stability of the restoration material, in recent years $[1,2]$. One of the most important criteria in evaluating the esthetic outcome of resin-based composite (RBC) restorations is surface roughness [3]. Surface characteristics and imperfections such as roughness or inadequate microhardness resulting from insufficient finishing and polishing procedures and polymerization remain as the main factors disrupting the color stability. Resin composite matrices absorb water to compensate polymerization shrinkage depending on the hydrolytic stability of the material $[2,4]$. Weakness in this stability leads to poor bonding of the material on the resin filler interface, deterioration of silanization, reduced 
surface hardness and reduced wear resistance. Insufficient polymerization results in the presence of unreacted free monomers causing increased solubility, microleakage and possibly discoloration due to the decrease in physical properties of the material $[4,5]$.

The discoloration capacity of the resin composites is mainly related to the polymerization level as well as type, size and shape of the inorganic filler particles of the material [6]. Water absorption due to insufficient polymerization and deficiencies in finishing and polishing procedures are the factors mainly caused by the errors in treatment protocol, resulting in surface porosities and color stability problems [7]. Polishability of the composite materials directly effects the optical properties, whereas also influencing the surface roughness of the restoration as well [8]. A smooth restoration surface reduces plaque accumulation, thus minimizing the possibility of periodontal diseases and caries process [9]. However, not only the material-related factors but also poor oral hygiene, smoking habits, eating habits of the patient may affect the discoloration of the restorations [10].

Discoloration may result from internal and external factors. An important internal factor reported to cause composite resins to change color over a long period was the oxidation of monomers or catalysts [11]. However, since the light-curing composite formulations do not contain benzoyl peroxide, the internal discoloration in these materials has been significantly reduced by time.

Surface covering agents (surface sealants) have been developed to reduce the discoloration capacity of the RBCs [9]. Surface sealants are low-viscosity, resinbased materials covering the surface of the composite restorations by penetrating through micro-structural defects [12]. These agents aim to reduce the water absorption of the material, thereby minimizing the discoloration of the resin composite by wrapping the surface of the restoration in a thin film layer [13]. Some researchers suggested the application of surface sealants, in terms of covering porosities, maintaining surface smoothness, increasing wear resistance, and ensuring the structural integrity of the RBC restoration $[11,13]$. However, there are also some studies that determined the use of surface sealants as controverseal in this respect [14]. There are various brands of surface sealants on the market, each containing different contents and having different application protocols.

Today, several diagnostic techniques, including shade guides, digital color measurement devices and macro dental photography, can be used for selecting the proper shade of the restoration material. However, not only the device, but also the quantitative scoring is required for the accurate longitudinal evaluations [15]. Quantitative scoring of the color alterations is generally performed according to the Comission Internationale de l'Eclairage (CIE) color space $[6,16,17,18,19]$. This color space includes mainly two systems including different color coordinates: $\mathrm{L}^{*} \mathrm{a}^{*} \mathrm{~b}^{*}$ and $\mathrm{L}^{*} \mathrm{C}^{*} \mathrm{~h}$. According to the CIELAB system, the color is expressed by three parameters: $L^{*}$ brightness between black $(0)$ and white $(100)$, color from $a^{*}$ red $\left(+a^{*}\right)$ to green $\left(-a^{*}\right), b^{*}$ refers to the color from yellow $\left(+b^{*}\right)$ to blue $\left(-b^{*}\right)[16,19]$. On the other hand, CIELCH color system is preferred by engineering experts as being more compatible with the way the human eye perceives color. This color space, unlikely $L^{*} a^{*} b^{*}$, uses cylindrical coordinates instead of triangular coordinates. $\mathrm{L}^{*}$ denotes luminance, $\mathrm{C}^{*}$ chroma, and $h^{*}$ denotes hue angle [18]. Also, a more sensitive color system, CIEDE2000 $\left(\Delta \mathrm{E}_{00}\right)$, was introduced based on CIELAB system, improving the performance of blue and gray colors $[6,20]$. There are controversial results in literature, reporting CIEDE2000 as a more sensitive system or not significantly different compared to the CIELAB [17,20].

Perceptibility threshold (PT) is defined as the smallest perceptible color difference that can be detected by an observer. $50: 50 \%$ perceptibility threshold expresses that $50 \%$ of the observers recognize a color difference between two objects while the other 50\% can't. Similarly, $50: 50 \%$ acceptability threshold (AT) indicates that $50 \%$ of the observers would consider color correction while the other $50 \%$ would consider it acceptable. Various threshold values for evaluating the color change have been reported previously, including $\Delta \mathrm{E} \geq 2.6$ [21], $\geq 3.3$ $[4,22]$, and $\geq 3.7[23,24,25]$ for perceptibility, and $\Delta \mathrm{E}<5.5$ $[21]$, and $<6.8[23,25]$ for clinical acceptability. The most recent accepted tresholds for tooth-colored materials were reported as follows: $\geq 1.2$ for perceptibility and $<2.7$ for acceptability [26].

The aim of this in vitro study was to quantitatively evaluate the effect of different surface sealants on color change of different resin composite materials, using a spectrophotometer. The tested null hypothesis $\left(h_{0}\right)$ was that the use of surface covering agents had no effect on the color stability of resin composites.

\section{Methods}

\section{Preparation and distribution of the samples}

A2 enamel shades of a nanofilled composite (Filtek Ultimate) and two nanohybrid composites (Charisma Topaz; Clearfil Majesty Es-2 Premium) were used for the preparation of samples (Table I). The compositions of the materials used were listed in table I. According to the power analysis, 40 disc-shaped composite samples in 12 $\mathrm{mm}$ in diameter and $2 \mathrm{~mm}$ in thickness were prepared, for each composite brand (Figure 1). The diameters of the samples were measured and checked by using a contact type digital micrometer (BMI 770150, Germany) for standardization. Regarding the production of the samples, a transparent matrix tape was placed on a mixing glass and a rubber seal was placed on the top. The resin composite 
was embedded inside the rubber seal and the top was covered with a transparent matrix tape and mixing glass again. A polywave LED curing unit (Valo Grand, Ultradent Products, USA) with $12 \mathrm{~mm}$ tip in diameter, was used for $20 \mathrm{~s}$ at $1000 \mathrm{mw} / \mathrm{cm}^{2}$ irradiation for the polymerization of each of the samples. The output of the curing unit was checked by using a led radiometer (SDI Limited, Victoria, Australia) before each use. All samples were polymerized from both sides to achieve standardized surface hardness before the staining procedure.

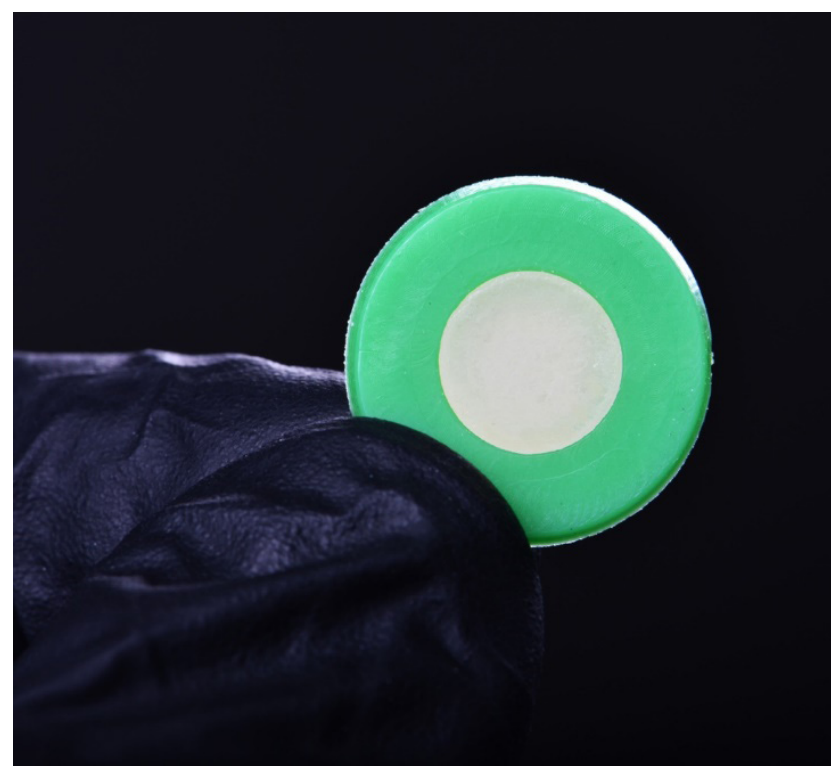

Figure 1. The composite sample in the silicone mold.
The samples were kept in distilled water at room temperature for one day before the baseline measurements. Then the surfaces of the samples were abraded manually by a single operator using 600 grit silicon carbide (SiC) abrasion papers $(30 \mu \mathrm{m}$ average particle size; Metkon Instruments Inc., Bursa, Turkey) for $20 \mathrm{~s}$ for initial surface standardization. A new abrasion paper was used for each sample. The abraded surfaces were gently cleaned from debris under continuous water and kept in distilled water before and during the color measurements.

The prepared samples $(n=120)$ were distributed to three composite groups (i.e., FU, CT, and CM). Each composite group was then divided into three experimental surface sealant groups and a control group (i.e., Permaseal, Biscover LV, Optiglaze groups, and control group).

Polishing and sealant application protocol

All samples were polished on both sides using Sof-Lex (3M, MN, USA) polishing discs in four different grains (thick, $80 \mu$; medium, $40 \mu$; fine, $24 \mu$; ultrafine, $8 \mu$ ), respectively. In order to perform this, the samples were fixed on an industrial clamp and $1.4 \mathrm{~N}$ load was also attached on the tip of the handpiece. No additional push-on force was used by the operator during the polishing procedures. In this wise, considering the weight of the handpiece as an additional load on the surface, an approximate constant load of $1.5 \mathrm{~N}$ was applied on the sample surface for standardization of polishing pressure. New discs were used for each sample to ensure the standardization. Each disc was used for $30 \mathrm{~s}$ with a handpiece rotating at $10.000 \mathrm{rpm}$, under constant water cooling to avoid heat generation.

Table I. Composition, type and manufacturer of the resin composites and the surface sealants.

\begin{tabular}{|c|c|c|c|c|c|}
\hline Code & Material & Shade & Filler & Composition & Manufacturer \\
\hline FU & $\begin{array}{l}\text { Filtek } \\
\text { Ultimate }\end{array}$ & $\begin{array}{l}\text { A2 } \\
\text { enamel }\end{array}$ & Nanofilled & $\begin{array}{l}\text { Matrix: Bis-GMA, UDMA, TEGDMA, PEGDMA, Bis-EMA } \\
\text { Filler: Silica filler }(20 \mathrm{~nm}) \text {, zirconia filler }(4-11 \mathrm{~nm}) \text {, zirconia/silica } \\
\text { cluster filler. } \\
0.6-10 \text { microns particle size. } 78.5 \mathrm{wt} \%, 63.5 \mathrm{vol} \%\end{array}$ & $3 \mathrm{M}, \mathrm{MN}, \mathrm{USA}$ \\
\hline $\mathrm{CM}$ & $\begin{array}{l}\text { Clearfil } \\
\text { Majesty ES-2 }\end{array}$ & $\begin{array}{l}\text { A2 } \\
\text { enamel }\end{array}$ & Nanohybrid & $\begin{array}{l}\text { Matrix: Bis-GMA, hydrophobic aromatic DMA, and hydrophobic } \\
\text { aliphatic DMA, dl-Camphorquinone } \\
\text { Filler: Silanated barium glass (average particle size } 0.7 \mathrm{~mm} \text { ) and pre- } \\
\text { polymerized organic filler. } 78 \mathrm{wt} \%, 66 \mathrm{vol} \%\end{array}$ & $\begin{array}{l}\text { Kuraray, Osaka, } \\
\text { Japan }\end{array}$ \\
\hline CT & $\begin{array}{l}\text { Charisma } \\
\text { Topaz }\end{array}$ & $\begin{array}{l}\text { A2 } \\
\text { enamel }\end{array}$ & Nanohybrid & $\begin{array}{l}\text { Matrix: TCD-DI-HEA } \\
\text { Filler: Barium aluminum fluoride glass filler of } 0.02-2 \mu \mathrm{m}, 5 \mathrm{vol} \% \\
\text { pyrogenic silicon dioxide filler of } 0.02-0.07 \mu \mathrm{m} .76 \mathrm{wt} \%, 65 \mathrm{vol} \%\end{array}$ & $\begin{array}{l}\text { Kulzer, Hanau, } \\
\text { Germany }\end{array}$ \\
\hline- & Permaseal & - & Unfilled & $\begin{array}{l}\text { Bis-GMA } 60 \% \text {, TEGDMA } 40 \% \text {, 1-dimethylaminoethyl metacrylate } \\
<3 \%\end{array}$ & $\begin{array}{l}\text { Ultradent Products, } \\
\text { UT, USA }\end{array}$ \\
\hline- & Biscover LV & - & Unfilled & Diğentaerythritol penta-acrylateesters and ethanol & Bisco, IL, USA \\
\hline- & $\begin{array}{l}\text { Optiglaze } \\
\text { Color Clear }\end{array}$ & - & Nanofilled & $\begin{array}{l}\text { Methyl-methacrylate (30-40\%), Silica filler(10\%), } \\
\text { Multifunctional acrylate(50-60\%), diphenyl(2,4,6-trimethylbenzoyl)- } \\
\text { phosphine oxide(less than5\%), Photoinitator }\end{array}$ & $\begin{array}{l}\text { GC Corp., Tokyo, } \\
\text { Japan }\end{array}$ \\
\hline
\end{tabular}


Following the polishing procedure, the polished surfaces were gently cleaned from debris. The three surface sealant agents (Table I) were applied to the polished surfaces, according to the instructions for use. The curing light was used for the polymerization of the surface sealants at an irradiation of $1000 \mathrm{mw} / \mathrm{cm}^{2}$. Regarding the Permaseal application, both surfaces of the polished samples in the first group were roughened with $37 \%$ phosphoric acid for $20 \mathrm{~s}$ to clean the surfaces. Subsequently Permaseal was applied on both surfaces, refined with slight air for $5 \mathrm{~s}$, and polymerized for $20 \mathrm{~s}$ per side. Regarding the Biscover LV application, both surfaces of the samples in the second group were roughened with $37 \%$ phosphoric acid for $30 \mathrm{~s}$. Then Biscover LV was applied on both surfaces, slightly refined with air, and polymerized for $20 \mathrm{~s}$ per side. Regarding the Optiglaze Color Clear application, both surfaces of the samples in the third group were roughened with hydrofluoric acid for $20 \mathrm{~s}$. Then, silanization was performed on both sides, as per manufacturers' instructions. Following that, Optiglaze Color Clear was applied on both surfaces, slightly refined with air, and polymerized for $40 \mathrm{~s}$ per side. Samples in the fourth group were not subjected to any surface sealant application after polishing and considered as the control group of the study.

Following the application of the surface sealants, the second color measurements were performed.

\section{Discoloration protocol}

In the present study, coffee solution was prepared daily as the staining solution by dissolving $20 \mathrm{gr}$ of coffee (Nescafe Gold, Nestle SA, Switzerland) in $250 \mathrm{ml}$ of $100{ }^{\circ} \mathrm{C}$ boiling water. The samples were kept in staining solution in a shaking incubator (Biobase Group, Meihua, China) at constant $37^{\circ} \mathrm{C}$ for total of 144 hours. The staining solution was renewed daily. After the discoloration process, the samples were rinsed with water for $10 \mathrm{~s}$ and gently dried before the final color measurements.

\section{Evaluation and statistical analysis}

All color measurements were performed by a single experienced researcher on a neutral gray background (CIE L* $=94.48, a^{*}=-0.16$, and $b^{*}=-0.21$ ) as referred in literature previously [27], using a clinical contact type spectrophotometer device (EasyShade 5, Vita-Zahnfabrik, Bad Säckingen, Germany). Spectrophotometer was calibrated before each use with the individual stand. The CIE $L^{*} C^{*} h *$ coordinates were recorded for each sample to evaluate the color changes. Readings were obtained in three different stages: baseline $\left(\mathrm{T}_{1}\right)$, following the sealant application $\left(\mathrm{T}_{2}\right)$, and after discoloration procedure $\left(\mathrm{T}_{3}\right)$.

Collected $\mathrm{L}^{*}, \mathrm{C}^{*}, \mathrm{~h}^{*}$ coordinates were converted to the corresponding $\mathrm{L}^{*}, \mathrm{a}^{*}, \mathrm{~b}^{*}$ coordinates for each sample using an online color converter software (http:// colormine.org/color-converter). Then the color changes between $\mathrm{T}_{2}-\mathrm{T} 1\left(\Delta \mathrm{E}_{1}\right), \mathrm{T}_{3}-\mathrm{T}_{2}\left(\Delta \mathrm{E}_{2}\right)$ and $\mathrm{T}_{3}-\mathrm{T}_{1}\left(\Delta \mathrm{E}_{3}\right)$ evaluation periods were calculated in terms of $\Delta \mathrm{E}^{*}$ values, using a special formula [1]:

$$
\Delta \mathrm{E} a b=\sqrt{(\Delta L)^{2}+(\Delta a)^{2}+(\Delta b)^{2}}
$$

The respective threshold $\Delta \mathrm{E}^{*}$ values for perceptibility and acceptibility were considered as $>1.2$ [26] and $\leq 2.7$ [26], respectively.

The data were analyzed with IBM SPSS V23. $\Delta \mathrm{E}^{*}$ values were analyzed with composite, sealant and evaluation period based generalized linear models. Results of the analysis were presented as mean \pm deviation. Intraclass correlation coefficients (ICCs) were obtained for correlations between the repeated measurements of the observer. Friedman test and Three-way Anova test were used for multiple comparisons within the groups. Intra-group binary comparisons of meaningful data were measured using Wilcoxon test and 'p' value of .05 was deemed significant.

\section{Results}

Generalized linear model results of the factors and interactions were listed in Table II. Composite, sealant, and $\Delta \mathrm{E}$ evaluation period were considered as effective factors influencing the color change. $\Delta \mathrm{E}$ evaluation period was considered the most effective factor $(\mathrm{p}<0.001)$, followed by composite factor $(\mathrm{p}<0.001)$ and sealant factor $(\mathrm{p}<$ $0.001)$, respectively (Table II).

Table II. Linear model analysis of composite, sealant, and $\Delta \mathrm{E}$ evaluation period factors.

\begin{tabular}{lccc} 
& Wald Chi-Square & df & Sig. \\
\hline Composite & 138.257 & 2 & $<0.001$ \\
Sealant & 65.744 & 3 & $<0.001$ \\
$\Delta$ E evaluation period & 326.513 & 2 & $<0.001$ \\
Composite * Sealant & 47.421 & 6 & $<0.001$ \\
Composite * $\Delta$ E evaluation period & 100.463 & 4 & $<0.001$ \\
Sealant * $\Delta$ E evaluation period & 37.22 & 6 & $<0.001$ \\
Composite * Sealant * $\Delta$ E evaluation period & 37.861 & 12 & $<0.001$
\end{tabular}


Table III. Three-way Anova comparisons of the selants, composites and the $\Delta \mathrm{E}$ evaluation period $\left(\Delta \mathrm{E}_{1}, \Delta \mathrm{E}_{2}\right.$, and $\left.\Delta \mathrm{E}_{3}\right)$.

\begin{tabular}{|c|c|c|c|c|c|c|}
\hline & \multicolumn{6}{|c|}{ Sealant } \\
\hline & Comp. & Permaseal & Biscover LV & Optiglaze & Control & Total \\
\hline \multirow{4}{*}{$\Delta \mathbf{E}_{1}$} & FU & $0.71 \pm 0.27 \underline{I}$ & $1.08 \pm 1.36^{\mathrm{I}}$ & $0.64 \pm 0.36^{\underline{I}}$ & $0.72 \pm 0.25^{\underline{I}}$ & $0.79 \pm 0.72^{B}$ \\
\hline & CT & $3.46 \pm 1.43 \stackrel{\mathrm{EFGHI}}{\mathrm{EFH}}$ & $2.62 \pm 1.68^{\mathrm{FGHI}}$ & $2.87 \pm 1.29 \underline{\mathrm{EFGHI}}$ & $1.93 \pm 0.92 \underline{\underline{\mathrm{GHI}}}$ & $2.72 \pm 1.42^{D}$ \\
\hline & $\mathbf{C M}$ & $0.63 \pm 0.33^{\underline{I}}$ & $1.04 \pm 1.05^{\mathrm{I}}$ & $1.06 \pm 0.49 \underline{I}$ & $1.51 \pm 0.91 \underline{\mathrm{HI}}$ & $1.06 \pm 0.79^{B}$ \\
\hline & Total & $1.60 \pm 1.57^{B}$ & $1.58 \pm 1.53^{B}$ & $1.52 \pm 1.26^{B}$ & $1.39 \pm 0.89^{B}$ & $1.52 \pm 1.33$ \\
\hline \multirow{4}{*}{$\Delta \mathbf{E}_{2}$} & FU & $3.82 \pm 2.07$ DEFGHI & $3.10 \pm 1.54^{\mathrm{EFGHI}}$ & $4.79 \pm 3.62$ СDEFGH & $2.64 \pm 1.91 \underline{\mathrm{FGHI}}$ & $3.59 \pm 2.47^{E}$ \\
\hline & CT & $7.46 \pm 3.00 \underline{\mathrm{ABC}}$ & $2.59 \pm 1.34 \stackrel{\mathrm{FGHI}}{ }$ & $1.83 \pm 0.59 \underline{\underline{\mathrm{GHI}}}$ & $5.98 \pm 1.82 \frac{\mathrm{BCDE}}{\underline{\mathrm{B}}}$ & $4.47 \pm 2.98^{C}$ \\
\hline & $\mathbf{C M}$ & $10.13 \pm 1.68^{\underline{A}}$ & $5.67 \pm 2.26 \underline{\mathrm{BCDEF}}$ & $7.00 \pm 3.28 \underline{\underline{\mathrm{ABCD}}}$ & $7.03 \pm 3.15^{\mathrm{ABCD}}$ & $7.46 \pm 3.06^{A}$ \\
\hline & Total & $7.14 \pm 3.45^{G}$ & $3.79 \pm 2.18^{D}$ & $4.54 \pm 3.49^{C}$ & $5.22 \pm 2.98^{A C}$ & $5.17 \pm 3.28$ \\
\hline \multirow{4}{*}{$\Delta \mathbf{E}_{3}$} & FU & $3.87 \pm 2.19$ DEFGHI & $2.57 \pm 1.22 \underline{\mathrm{FGHI}}$ & $5.01 \pm 3.48 \underline{\underline{\text { CDEFG }}}$ & $2.79 \pm 1.81 \stackrel{\text { EFGHI }}{ }$ & $3.56 \pm 2.44^{E}$ \\
\hline & $\mathbf{C T}$ & $6.04 \pm 2.78 \underline{\mathrm{BCDE}}$ & $2.95 \pm 1.54$ EFGHI & $3.18 \pm 0.79 \underline{\underline{\text { EFGH }}}$ & $6.05 \pm 2.03 \underline{\mathrm{BCDE}}$ & $4.55 \pm 2.39^{c}$ \\
\hline & $\mathbf{C M}$ & $10.16 \pm 1.31^{\mathrm{A}}$ & $5.04 \pm 2.33 \frac{\mathrm{CDEFG}}{\mathrm{C}}$ & $7.33 \pm 3.37 \underline{\mathrm{ABC}}$ & $8.60 \pm 1.37 \underline{\mathrm{AB}}$ & $7.78 \pm 2.88^{A}$ \\
\hline & Total & $6.69 \pm 3.39^{F G}$ & $3.52 \pm 2.03^{D}$ & $5.17 \pm 3.23^{A C}$ & $5.81 \pm 2.96^{A F}$ & $5.30 \pm 3.14$ \\
\hline \multirow{4}{*}{ Total } & FU & $2.80 \pm 2.26^{E F}$ & $2.25 \pm 1.59^{E}$ & $3.48 \pm 3.47^{F G}$ & $2.05 \pm 1.76^{E}$ & $2.65 \pm 2.42^{\mathrm{a}}$ \\
\hline & CT & $5.65 \pm 2.94^{A}$ & $2.72 \pm 1.48^{E F}$ & $2.62 \pm 1.08^{E F}$ & $4.66 \pm 2.53^{B D}$ & $3.91 \pm 2.48^{\mathrm{b}}$ \\
\hline & $\mathbf{C M}$ & $6.97 \pm 4.72^{C}$ & $3.91 \pm 2.82^{B G}$ & $5.13 \pm 3.94^{A D}$ & $5.71 \pm 3.67^{A}$ & $5.43 \pm 3.96^{c}$ \\
\hline & Total & $5.14 \pm 3.85^{\mathrm{a}}$ & $2.96 \pm 2.15^{b}$ & $3.74 \pm 3.23^{c}$ & $4.14 \pm 3.14^{\mathrm{c}}$ & $4.00 \pm 3.24$ \\
\hline
\end{tabular}

$\Delta \mathbf{E}_{1}$ : Color change between sealant application and baseline; $\Delta \mathbf{E}_{2}$ : Color change between discoloration and sealant application; $\Delta \mathbf{E}_{3}:$ Color change between discoloration and baseline; a-c: Regardless of the evaluation period, no significant difference was found between the sealants with the same letter; $\boldsymbol{A}-\boldsymbol{G}$ : No significant difference was found between the composites and between the sealants with the same

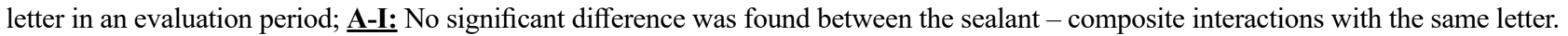

In this study, kappa values of 0.94 and 0.91 were obtained for intra-observer agreement, during the spectrophotometric measurements in pilot study, according to the ICC results. As a result of considering a very good and positive agreement between the measurements of two operators, the study was initiated with operator 1 .

The $\Delta \mathrm{E}$ values ranged from $0.63 \pm 0.33$ to $10.16 \pm$ 1.31. The three-way multiple comparisons of the selant agents, resin composites and the $\Delta \mathrm{E}$ values were listed in table III.

\section{Assessment of the surface sealants}

There were no statistically significant differences among the sealants, regarding $\Delta \mathrm{E}_{1}$ values $(\mathrm{p} \geq 0.05)$, and all were below AT $(\leq 2.7)$, but above PT $(>1.2)$ color changes. Higher and statistically significant $\Delta \mathrm{E}^{*}$ values were observed for all groups regarding the $\Delta \mathrm{E}_{2}$ values compared to the $\Delta \mathrm{E}_{1}$ values $(\mathrm{p}<0.001) . \Delta \mathrm{E}_{2}$ and $\Delta \mathrm{E}_{3}$ values were above $\mathrm{AT}(>$ 2.7) for all sealant groups (Table III).

Regardless of the composite brands and the evaluation periods, the amount of color change for Biscover LV was the lowest among all sealants $(p<0.001)$. The highest color change was observed for Permaseal $(p<0.001)$. The color change for Optiglaze and control groups were statistically similar $(\mathrm{p} \geq 0.05)$. All the sealants presented unacceptable amount of color change (Table III).

\section{Assessment of the resin composites}

Regarding $\Delta \mathrm{E}_{1}$ values, the color change for $\mathrm{CT}$ composite was significantly higher among all $(p<0.001)$, and there was no significant difference between FU and CM $(\mathrm{p} \geq 0.05)$. The color changes of the FU and CM composites were also below PT $(\leq 1.2)$, whereas it was above AT $(>$ 2.7) for $\mathrm{CT}$ composite. Regarding the $\Delta \mathrm{E}_{2}$ and $\Delta \mathrm{E}_{3}$ values, all composites presented unacceptable color changes. Additionally, FU presented significantly the lowest amount of color change for both $\Delta \mathrm{E}_{2}$ and $\Delta \mathrm{E}_{3}$ values $(\mathrm{p}=0.032, \mathrm{p}=$ 0.016 , respectively; Table III).

Regardless of the sealant groups and the evaluation periods, significantly the lowest $\Delta \mathrm{E}$ was determined for $\mathrm{FU}$ composite, followed by CT and CM $(\mathrm{p}<0.001)$. The color change was below AT $(\leq 2.7)$ only for the FU composite.

\section{Assessment of composite / sealant interactions}

Regardless of the evaluaton periods, $\Delta \mathrm{E}$ values for FU - Biscover LV and CT - Optiglaze combinations were determined to have the lowest and the only acceptable color changes among all. CM - Permaseal combination presented significantly the highest amount of change $(\mathrm{p}<0.001)$, followed by CT - Permaseal and CM - Optiglaze.

$L^{*}, a^{*}, b^{*}$ parameters were also evaluated by performing three repeated measurements. The parameters were analyzed individually for the sealant groups, to evaluate the individual effect of color parameters on color changes. $\mathrm{L}^{*}$ and $\mathrm{b}^{*}$ parameters decreased mathematically during the overall evaluation period, however the differences were not statistically significant $(p \geq 0.05)$. Only the decreases in $a^{*}$ parameter were found statistically significant $(\mathrm{p}<0.001)$. 


\section{Discussion}

The surface covering agents have influenced the color stability of the resin-based composite materials, according to the results of the present study. However, this effect was either positive or negative, and the level of the effect was sealant and composite type dependent. Therefore, the null hypothesis $\left(\mathrm{h}_{0}\right)$ of this study was rejected.

In the present study, the samples were pre-polished using the gold standard polishing instruments, flexible aluminum oxide discs, to obtain the standardization and to increase the effectiveness of the surface sealants, thereby providing more accurate color determinations by minimizing the micro-gap formations on the surfaces $[13,22,28]$. Moreover, in order to mimic the daily routine in vitro, coffee was used as one of the most effective staining agents $[28,29]$. The discoloration mechanism of coffee was explained by adsorbtion and absorbtion of the yellow colorants through the organic phase of the resinbased composites [29]. Guler et al. [30] and Rajkumar et al. [31] mentioned $15 \mathrm{~min}$ as the average time for consumption of a cup of coffee and 3.2 cups as the average consumption per day [32]. Also, 72 hours of simulated coffee consumption was considered as correspondence to 3 months of daily consumption by Rajkumar et al [31]. Mundim et al. reported that, 15 days of storage in coffee, simulated consumption of the drink over one year [5]. Moreover, hot coffee solution was determined as a more active agent for discoloration previously [31]. Hui et al. reported the degree of discoloration was proportional to the increase of the temperature for composite samples which surface sealant was applied on [33]. In order to simulate the oral conditions, $37^{\circ} \mathrm{C}$ temperature and constant exposure to staining solution were suggested [13]. Regarding these facts, in the present study, the samples were kept in coffee solution at constant $37{ }^{\circ} \mathrm{C}$ in an incubator for 144 hours, which was corresponding to a person's coffee consumption of 6 months [30,31]. The solution was renewed daily.

In this in vitro study, the most commonly accepted $\Delta \mathrm{E}$ threshold values of $>1.2$ and $\leq 2.7$ were considered for PT and AT of color change, respectively. The color of resin composites have been invesitigated for years, however, there has been no consensus on the limits of PT and AT. O'Brien et al. determined that values of 2 or less were clinically acceptable [7]. Vichi et al. [4] and Tuncer et al. [22] reported value of 3.3 as perceptible, whereas Kim and Lee [24] and Celik et al. [34] determined 3.7. Johnston and Kao [23] reported $\Delta \mathrm{E}^{*}$ threshold values of 3.7 and 6.8 , and Douglas et al. [21] 2.6 and 5.5 for perceptibility and acceptability, respectively among dentists. Most recently, Paravina et al. published a comprehensive review of clinical and research applications in dentistry, and considered $>1.2$ for PT and $\leq 2.7$ for AT for tooth-colored materials [26]. In order to obtain a complete color stability $(\Delta \mathrm{E}=0)$ for a material, no color difference should be detected after being exposured to testing environment [35].
This study used a common color system CIELAB $\Delta \mathrm{E}$, by converting the collected $\mathrm{L}^{*}, \mathrm{C}^{*}, \mathrm{~h}^{*}$ coordinates to corresponding $L^{*}, a^{*}, b^{*}$ coordinates for each sample, to assess the color alterations. This color system was selected for a better comparison with previous studies $[6,17,22,34]$. Readings of $L^{*}$ and $b^{*}$ parameters decreased mathematically for all evaluation periods, but these differences were not statistically significant. However, the decreases in $a^{*}$ parameter were found significant, therefore, the amount of color changes regarding $\Delta \mathrm{E}$ values were primarily due to the changes in $\mathrm{a}^{*}$ parameter.

Composite, sealant and $\Delta \mathrm{E}^{*}$ evaluation period were all determined as effective factors to influence the color change in this study ( $p<0.001$ for each factor). However, sealant variable was considered less effective compared to the composite variable (Table II).

\section{Assessment of the resin composites}

A nanofilled composite FU presented acceptable and significantly the lowest amount of color change, whereas a nanohybrid CM composite presented the highest (Table III). This result was similar to the results of Tuncer et al [22]. Nasim et al. [36] reported, less color change might be expected for nanofilled composites, as having smoother surfaces with less stains, due to smaller particle sizes. Also, Reddy et al. reported that, nanofilled composites to have less color change than hybrid-composites [37]. Similar inorganic filler concentrations of the composites (FU: 78.5 wt $\%$, 63.5 vol $\%$; CT: $76 \mathrm{wt} \%, 65 \mathrm{vol} \%$; CM: $78 \mathrm{wt} \%$, $66 \mathrm{vol} \%$ ) could not be the explanation of this difference. However, the type of the fillers (silica, zirconia and silica/ zirconia cluster fillers), as well as the size of the fillers in FU composite might have influenced the color stability $[9,22]$. Another possible explanation is that triethyleneglycol dimethacrylate (TEGDMA) in FU might have enhanced the surface hardness, elastic modulus and the degree of polymerization, thus contributed to better resistance against discoloration $[9,38]$. On the other hand, TEGDMA containing FU might have had the disadvantage of being significantly sensitive to discoloration when immersed in hot coffee compared to cold [31]. As mentioned previously, water absorption affects the mechanical properties of composites toward hydrolytic degradation, and as the proportion of TEGDMA increased from $0 \%$ to $1 \%$, the water absorption of hydrophilic bisphenol-glycidyl methacrylate (Bis-GMA) based resins were reported to be increased form $3 \%$ to $6 \%[22,28]$. Whereas, FU also contains a more stain resistant monomer urethane dimethacrylate (UDMA), which was determined to have lower rate of water absorption $[28,31]$. This monomer might have had a supporting effect that inhibited the possible adverse effects of TEGDMA.

The color change of CT composite was significantly higher than the other nanohybrid CM composite, in the present study (Table III). Bis-acryloyloxymethyl tricyclo decan (TCD-DI-HEA) monomer in CT composite was 
reported to have important affinity to low polarity colorants of coffee, thus this monomer might be the reason of low color stability [30]. The color stability of restorative materials was determined to change not only depending on the resin matrix, filler type, size and concentration, but also the minor pigments, types and concentrations of the initiators, inhibitors and activators and oxidation of unreacted carbon bonds [19,22]. These factors might have also influenced the color stability of the composites tested in this study.

\section{Assessment of the surface sealants}

In this study, all surface sealants presented color changes above the AT. The level of color changes in experimental sealants were lower than the control group, except the permaseal group. This result partially supported the results of Pedroso et al., who reported lower degree of discoloration for the composite samples with surface sealant [12]. But in the present study, this outcome was sealant type and also composite type dependent.

Biscover LV have supported the color stability and the lowest amount of color changes were recorded for the Biscover LV group in our study. Opposing to our result, Gurbuz et al. reported Biscover LV as having no significant effect on the surface roughness [30]. Also, Sahin et al. indicated dipentaerythritolpentaacrylate-containing Biscover sealant as a less stain resistant agent compared to Optiglaze [39]. The reason for the lesser stain resistance was shown as the shorter polymerization time of Biscover, which was supporting the previous report of Doray et al [40]. The different outcomes might also be related to the use of these surface sealants on acrylic dentures. Curingtime was also not considered an effective factor according to our results. Although Optiglaze was polymerized twice (40 s) as much of Biscover LV (20 s) and Permaseal (20 s) in the present study, the lowest amount of color change was detected for Biscover LV rather than Optiglaze.

The highest amount of color change was detected for Permaseal group, which was also higher than the color changes of control group. This might be interpreted that, the use of Biscover LV inhibited the level of discoloration, however Permaseal affected the color stability of the resin composites adversely. Bis-GMA monomer in Permaseal might have been responsible for the the highest amount of discoloration, as this hydrophilic monomer was reported to be more prone to staining compared to UDMA $[12,28,39]$. Also, TEGDMA might have enhanced the water uptake of Bis-GMA-based Permaseal, as it was reported to have higher staining because of hydrophilicity and higher affinity to get discolored in hot coffee solutions [28,31]. Supporting this result, Corcodel et al. stated that surface sealant applications might have some adverse effects on color stability of resin-based restorative materials and clinicians should consider this situation [41].

The amount of color changes of Optiglaze and control groups were not statistically different. Therefore,
Optiglaze sealant had no significant effect on the color stability of the resin composites used, supporting the results of Cortopassi et al. who applied different surface sealants to the same type of composite samples and concluded that, the surface sealant application did not provide any benefit in terms of color stability [42]. Nanofillers in Optiglaze might have contributed to the surface discoloration in terms of this outcome as long as the debonding of inorganic fillers form the resin matrix might have formed void formations on surface and incresed the surface roughness [28]. Although there are also evidences that nanofillers might have positive effects on color stability, highly discolored Permaseal and relatively ineffective Optiglaze sealants might have had debonding problems, as they were applied on less retentive, well-polished composite surfaces and also were discolored in hot coffee solution [31,36].

\section{Assessment of composite / sealant interactions}

Khalaj et al. reported higher level of discoloration on nanohybrid composites was found for the samples with Permaseal surface sealant compared to the control group [1]. This result was similar to the results of our study. On the other hand, Pedroso et al. reported that, the combination of nanofilled composites with Biscover LV showed less discoloration among the experimental groups [12]. Also, Miotti et al. supported that with similar results of Biscover LV and microhybrid resin combination [10]. Our results have contributed these findings with lower amount of color change of Biscover LV sealant in combinations with both nanohybrid and nanofilled composites. The combination of nanofilled FU composite with Biscover LV also presented the lowest amount of color change among all, in accordance with Pedroso et al [12].

The combinations of FU - Biscover LV, CT - Biscover LV, and CT - Optiglaze were considered the most effective combinations for color stability in the present study (Table III). However, FU composite also presented a high rate of color stability without use of any surface sealant in our study. Therefore, it might be interpreted that, the use of surface sealants may not be advantageous every time. The superiority of the combinations with FU composite might be as a result of previously reported high surface polishing properties, with respect to the fact that positive effect of surface sealants was considered increasing when applied on well-polished surfaces [30,41,43]. Moreover, all of the combinations with $\mathrm{CM}$ composite and the combination of CT composite with Permaseal might be considered risky regarding the color stability.

This in vitro study also has some limitations. Only one type of staining solution was used to simulate the intraoral conditions. However, many other factors such as dietary habits, occlusal relations, the effect of saliva, tooth brushing, and mouth rinsing should be taken into consideration. It should be considered that different strengths of staining solutions or longer exposure times of the samples in the solutions might also influence the 
color changes. In terms of sealing ability, penetrability, and viscosity of the sealants might also be considered besides composition [13]. The conclusions of this study should be verified with further clinical trials.

\section{Conclusion}

Within the limitations of this study, regarding hot coffee discoloration for 6 months, the experimented surface covering agents might be determined effective on the color stability of resin composites. Biscover LV sealant have inhibited the level of discoloration, whereas Permaseal sealant had an increasing effect. Nanofilled FU composite might be determined to have the highest color stability, whereas nanohybrid CM composite had the lowest, regarding the coffee discoloration for 6 months. The combination of FU composite with Biscover LV, and CT composite with Optiglaze and Biscover LV might be considered as the most effective combinations for inhibiting the composite surface discoloration.

\section{References}

1. Joiner A, Luo W. Tooth colour and whiteness: A review. J Dent. 2017;67S:S3-S10.

2. Khalaj K, Soudi A, Tayefi-Nasrabadi M, Keshvad MA. The evaluation of surface sealants' effect on the color stability of Nano-hybrid composite after polishing with One-Step system (in-vitro). J Clin Exp Dent. 2018;10:e927-e932.

3. Beltrami R, Ceci M, De Pani G, Vialba L, Federico R, Poggio C, et al. Effect of different surface finishing/polishing procedures on color stability of esthetic restorative materials: A spectrophotometric evaluation. Eur J Dent. 2018;12:4956.

4. Vichi A, Ferrari M, Davidson CL. Color and opacity variations in three different resin-based composite products after water aging. Dent Mater. 2004;20:530-534.

5. Mundim FM, Garcia Lda F, Pires-de-Souza Fde C. Effect of staining solutions and repolishing on color stability of direct composites. J Appl Oral Sci. 2010;18:249-254.

6. de Oliveira DC, Ayres AP, Rocha MG, Giannini M, Puppin Rontani RM, Ferracane JL, et al. Effect of different in vitro aging methods on color stability of a dental resin-based composite using CIELAB and CIEDE2000 color-difference formulas. J Esthet Restor Dent. 2015;27:322-330.

7. Poggio C, Ceci M, Beltrami R, Mirando M, Wassim J, Colombo M. Color stability of esthetic restorative materials: a spectrophotometric analysis. Acta Biomater Odontol Scand. 2016;2:95-101.

8. Halacoglu DM, Yamanel K, Basaran S, Tuncer D, Celik C. Effects of staining and bleaching on a nanohybrid composite with or without surface sealant. Eur J Dent. 2016;10:361365 .

9. Nikolaidis A, Vouzara T, Koulaouzidou E. Pit and fissure nanocomposite sealants reinforced with organically modified montmorillonite: A study of their mechanical properties, surface roughness and color stability. Dent Mater J.
2020;39:773-783.

10. Miotti LL, Nicoloso GF, Durand LB, Susin AH, Rocha RO. Color stability of a resin composite: Effect of the immersion method and surface treatments. Indian $\mathrm{J}$ Dent Res. 2016;27:195-199.

11. Ruschel VC, Bona VS, Baratieri LN, Maia HP. Effect of Surface Sealants and Polishing Time on Composite Surface Roughness and Microhardness. Oper Dent. 2018;43:408415.

12. Pedroso LB, Barreto LF, Miotti LL, Nicoloso GF, Durand LB. Effect of a surface sealant on the color stability of composite resins after immersion in staining solution. Gen Dent. 2016;64:e22-e25.

13. Hepdeniz OK, Temel UB, Ugurlu M, Koskan O. The effect of surface sealants with different filler content on microleakage of Class V resin composite restorations. Eur J Dent. 2016;10:163-169.

14. Tekçe N, Pala K, Tuncer S, Demirci M. The effect of surface sealant application and accelerated aging on posterior restorative surfaces: An SEM and AFM study. Dent Mater J. 2017;36:182-189.

15. Da Silva JD, Park SE, Weber HP, Ishikawa-Nagai S. Clinical performance of a newly developed spectrophotometric system on tooth color reproduction. J Prosthet Dent. 2008;99:361-368.

16. Ghinea R, Pecho O, Herrera LJ, Ionescu AM, Cardona Jde L, Sanchez MP, et al. Predictive algorithms for determination of reflectance data from quantity of pigments within experimental dental resin composites. Biomed Eng Online. 2015; 14 Suppl 2 (Suppl 2):S4.

17. He WH, Park CJ, Byun S, Tan D, Lin CY, Chee W. Evaluating the relationship between tooth color and enamel thickness, using twin flash photography, cross-polarization photography, and spectrophotometer. J Esthet Restor Dent. 2020;32:91-101.

18. Pustina-Krasniqi T, Shala K, Staka G, Bicaj T, Ahmedi E, Dula L. Lightness, chroma, and hue distributions in natural teeth measured by a spectrophotometer. Eur J Dent. 2017;11:36-40.

19. Spina DR, Grossi JR, Cunali RS, Baratto Filho F, da Cunha LF, Gonzaga CC, et al. Evaluation of Discoloration Removal by Polishing Resin Composites Submitted to Staining in Different Drink Solutions. Int Sch Res Notices. 2015;2015:853975.

20. Lee YK. Comparison of CIELAB DeltaE* and CIEDE2000 color-differences after polymerization and thermocycling of resin composites. Dent Mater. 2005;21:678-682.

21. Douglas RD, Steinhauer TJ, Wee AG. Intraoral determination of the tolerance of dentists for perceptibility and acceptibility of shade mismatch. J Prosthet Dent. 2007;97:200-208.

22. Tuncer S, Demirci M, Tiryaki M, Ünlü N, Uysal Ö. The effect of a modeling resin and thermocycling on the surface hardness, roughness, and color of different resin composites. J Esthet Restor Dent. 2013;25:404-419.

23. Johnston WM, Kao EC. Assessment of appearance match by visual observation and clinical colorimetry. J Dent Res. 1989;68:819-822. 
24. Kim BJ, Lee YK. Influence of the shade designation on the color difference between the same shade-designated resin composites by the brand. Dent Mater. 2009;25:1148-1154.

25. Zimmerli B, Koch T, Flury S, Lussi A. The influence of toothbrushing and coffee staining on different composite surface coatings. Clin Oral Investig. 2012;16:469-479.

26. Paravina RD, Pérez MM, Ghinea R. Acceptability and perceptibility thresholds in dentistry: A comprehensive review of clinical and research applications. J Esthet Restor Dent. 2019;31:103-112.

27. Shokry TE, Shen C, Elhosary MM, Elkhodary AM. Effect of core and veneer thicknesses on the color parameters of two all-ceramic systems. J Prosthet Dent. 2006;95:124-129.

28. Barutcigil Ç, Yıldız M. Intrinsic and extrinsic discoloration of dimethacrylate and silorane based composites. J Dent. 2012;40 Suppl 1:e57-e63.

29. Bagheri R, Burrow MF, Tyas M. Influence of food-simulating solutions and surface finish on susceptibility to staining of aesthetic restorative materials. J Dent. 2005;33:389-398.

30. Guler AU, Yilmaz F, Kulunk T, Guler E, Kurt S. Effects of different drinks on stainability of resin composite provisional restorative materials. J Prosthet Dent. 2005;94:118-124.

31. Rajkumar K, Kumar S, Mahalaxmi S, Ragavi P, Mageshwaran TA. Colour stability of resin composites after emersing in coffee of different temperature - an in vitro study. SRM Univ J Dent Sci. 2011;2:92-5.

32. Anila Namboodiripad P, Kori S. Can coffee prevent caries? J Conserv Dent. 2009;12:17-21.

33. Hui R, Choi IH, Hussein I, Hockey J, Hetrelizides D, Wong RHK. The effect of drinks and temperature on the staining of resin composites coated with surface sealants. J Dent Biomater. 2014;1:16-22.

34. Çelik EU, Aladağ A, Türkün LŞ, Yilmaz G. Color changes of dental resin composites before and after polymerization and storage in water. J Esthet Restor Dent. 2011;23:179-188.

35. Tekçe N, Tuncer S, Demirci M, Serim ME, Baydemir C. The effect of different drinks on the color stability of different restorative materials after one month. Restor Dent Endod. 2015;40:255-261.

36. Nasim I, Neelakantan P, Sujeer R, Subbarao CV. Color stability of microfilled, microhybrid and nanocomposite resins--an in vitro study. J Dent. 2010;38 Suppl 2:e137-e142.

37. Reddy PS, Tejaswi KL, Shetty S, Annapoorna BM, Pujari SC, Thippeswamy HM. Effects of commonly consumed beverages on surface roughness and color stability of the nano, microhybrid and hybrid composite resins: an in vitro study. J Contemp Dent Pract. 2013;14:718-723.

38. Gurbuz O, Cilingir A, Dikmen B, Ozsoy A, Mert Eren M. Effect of surface sealant on the surface roughness of different composites and evaluation of their microhardness. Eur Oral Res. 2020;54:1-8.

39. Şahin O, Dede DÖ, Köroğlu A, Yılmaz B. Influence of surface sealant agents on the surface roughness and color stability of artificial teeth. J Prosthet Dent. 2015;114:130137.

40. Doray PG, Eldiwany MS, Powers JM. Effect of resin surface sealers on improvement of stain resistance for a composite provisional material. J Esthet Restor Dent. 2003;15:244-249; discussion 249-250.

41. Corcodel N, Hassel AJ, Sen S, Saure D, Rammelsberg P, Lux CJ, et al. Effects of staining and polishing on different types of enamel surface sealants. J Esthet Restor Dent. 2018;30:580-586.

42. Cortopassi LS, Shimokawa CAK, Willers AE, Sobral MAP. Surface roughness and color stability of surface sealants and adhesive systems applied over a resin-based composite. J Esthet Restor Dent. 2020;32:64-72.

43. Bolat M, Pancu G, S, Stoleriu S, Iovan G, Andrian S Comparative Study Regarding sourface roughness of different types of composite resins after finishing and polishing methods and glaze material application. Rom J Oral Rehabil. 2016;8:100-105. 